\title{
"The Missing Link": a Case Study of Sensing-and-Modeling Toolkits for Constructionist Scientific Investigation
}

\author{
Paulo Blikstein, Uri Wilensky \\ Center for Connected Learning and Computer-Based Modeling - Northwestern University \\ 2120 Campus Drive - Evanston, IL, USA - 60208 - tel. +1 (847) 491-5666 \\ [paulo,uri]@northwestern.edu
}

\begin{abstract}
Multi-agent modeling has been successfully used in several scientific fields, oftentimes transforming scientists' practice and mindsets. Educational researchers have also realized the potential of this modeling approach for learning. Studies have suggested that students are able to understand concepts above their expected grade level after interacting with curricula developed using multi-agent simulation. However, most multi-agent models are exclusively 'on-screen', without connection to the physical world. Real-time model validation and realworld sensing are very challenging to accomplish with extant modeling platforms. As an attempt to address this issue, we designed a technological platform to enable students to seamlessly connect multi-agent models and electronic sensors, in real time. The platform is designed for learners to validate, refine, and debug their computer models using real-world data. This paper focuses on the technical and pedagogical aspects of this project, describing pilot studies which suggest a real-to-virtual reciprocity that catalyzes further inquiry toward deeper understanding.
\end{abstract}

\section{Introduction}

A promising path to improve education using new technologies has been to bring the most advanced tools from research labs and adapt them for use in schools. Computer programming is probably the better-known case. The LOGO computer language, proposed by Seymour Papert [1] almost forty years ago, encapsulated the most powerful ideas in Computer Science at the time and made it available for children. The same happened to robotics in the late nineties [2]. With the introduction of robotics kits such as the LEGO Mindstorms kit, there has been an explosion of new learning opportunities for children of all ages, an unthinkable fact just some years before, when robotics was only researched in advanced laboratories in engineering schools. Mechanical advantage, gearing, mechanism design, data sensing, control, and feedback are just some examples of the powerful ideas made available to learners.

Multi-agent modeling and simulation, too, went through a similar path. Multi-agent methods have been used with great success in fields such as biology, sociology, chemistry, physics, economics, psychology, and engineering. They are dramatically changing scientists' mindsets and practice. Instead of departing from often very complex "aggregate" behaviors, scientists started to use massive computation power to simulate systems with thousands of very simple agents, behaving accordingly to simple rules. For example, instead of building an exact computer model of the known Gas Laws (for example, $P V=n R T$ ), an agentbased simulation would just attribute simple behaviors to the individual atoms in the system ("move", "collide", "bounce off walls"). The Gas Laws would then emerge from these simple rules. After Wilensky and Resnick [3] incorporated this powerful concept into learning-focused modeling tools, in the midnineties, a technique before restricted to supercomputing laboratories entered the classroom. Powerful ideas such as emergence, self-organization, and randomness were put in the hands (and minds) of children, and research has suggested important learning gains after interaction with curricula developed using multi-agent simulation $[4,5]$.

This paper describes a research agenda which attempts to find the "missing link" between these two last traditions, thus merging robotics/sensing and multi-agent computer simulation. Traditional computer modeling environments do not communicate with the world, and educational robotics aims to construct autonomous devices, with local, limited processing power. Moreover, since multi-agent simulation departs from simple rules to generate complex behaviors, data sensing could potentially be much simpler: instead of complex sensors, students could just detect simple physical interactions between the agents. 
The platform we designed enable learners to connect virtual and physical models as to validate, refine, and debug their computer models using realworld data. We will present proof-of-concept models that demonstrate the potential of such approach, as well as the learning benefits of this design framework, as suggested by our preliminary user studies.

\section{The quest for the missing link}

Despite their compartmentalization in the traditional school curriculum, Physics, Chemistry, and Biology are 'out-there in the world', entangled in a complex web of phenomena. Most are invisible to human vision and time scale. Many patterns in nature are too long, too fast or too small for learners to extract and understand the underlying structures. Canonical examples are weather behavior, chemical reactions, housing and traffic patterns, particle physics, and population ecology. Conventional school laboratories are not well equipped to support students in developing hypothesis about the information they gather. For example, a student studying a chemical reaction in a Chemistry laboratory might discern the components and even hypothesize as to the relations between them; however, the investigation cannot go much further. Later, in the classroom, he will learn about equations and theories which bear little resemblance to the phenomenon observed in the laboratory. Needed are tools that provide continuity between observation and model-building, providing the 'missing link' between data-gathering and the construction of theories using computational representations. That is, to make the study of these phenomena accessible to students, we need new technological tools that foreground and unveil the deep structures, in the virtual and physical world.

\section{The technological platform}

The typical activity of our pilot studies was for students to build, using the NetLogo [6] modeling-andsimulation environment, a computer algorithm of a particular scientific phenomenon, such as heat transfer or gas laws, and a physical apparatus equipped with electronic sensors. We developed special software components to link the models in real time through an open-source, low cost analog-to-digital interface - the GoGo Board [7]. Then, learners would create a computer interface to visualize outcomes side-by-side (Figure 1), comparing their results, and debugging their algorithm until it matches adequately the real-world data.

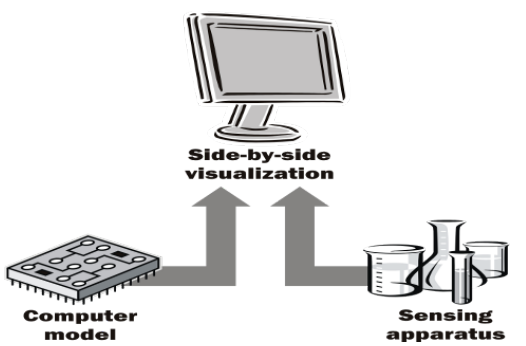

Figure 1 - Basic architecture of a trans-media system

We built proof-of-concept systems for trans-media explorations in heat transfer, gas laws, chemical reactions, and Materials Science. Figure 1 shows a model to investigate heat transfer using a multi-agent approach. Each cell in the hexagonal grid is an agent. The physical counterpart is a grid of 19 hexagonal cells and a lid with temperature sensors. Cells are filled with liquid at different temperatures. The sensors are connected to the analog-to-digital interface, and the data are fed directly into the computer model. The second example model investigates gas laws using pressure, temperature and volume sensors. As the volume of the syringe changes, the computer model varies accordingly, and students evaluate the match between sensor values and the results supplied by their own algorithms.

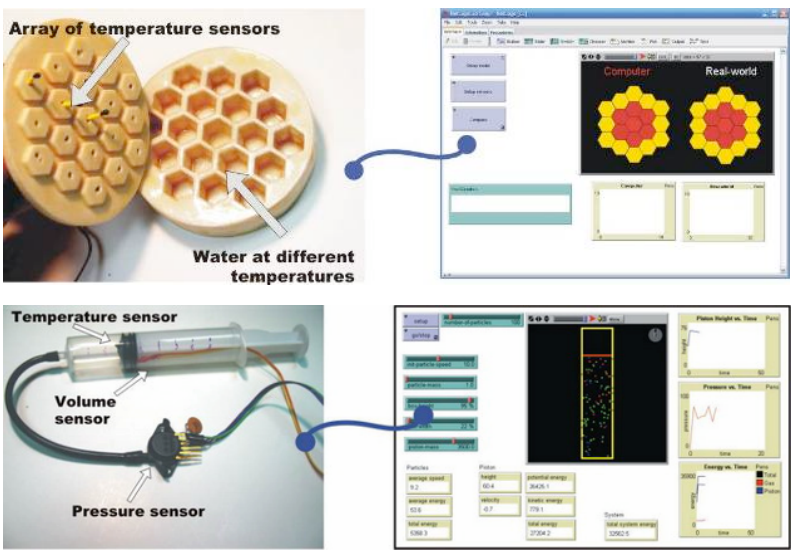

Figure 2. Two proof-of-concept trans-media models: heat transfer and gas laws.

\section{Pilot user studies}

In two pilot studies conducted in 2005 and 2006, we compared artifacts generated by undergraduate and graduate students under two distinct conditions. In the first one, students created purely virtual multi-agent models. In the second, students built models with sensors. All students built their models as an assignment in an 'Educational Design' course. Over two years, we had 14 participants (two groups of 
seven), approximately equally divided into the two groups. Our data include students' artifacts, field notes and transcriptions of post-interviews.

Preliminary results suggest that students belonging to the second group (virtual + physical modeling) attended to phenomenal factors which they would otherwise have overlooked (as they were not mentioned by students in the first group), such as energy loss, reversibility, and precision. Some new problem-solving avenues were explored: a group designing a sensor-equipped American Sign Language recognition glove was struggling to write a flexible and reliable code for gesture recognition. They ended up realizing that for such a problem it would be far more efficient to write a program to enable each user to train the system with real-world data from actual gestures. In a project for studying earthquake wave patterns, learners analyzed the propagation of multiple waves in a gelatin model they built, which helped them realize many of the bugs and limitations of the previously designed wave propagation algorithm. Similar statements were made by learners who built systems to study tsunami wave propagation and a forest fire spread.

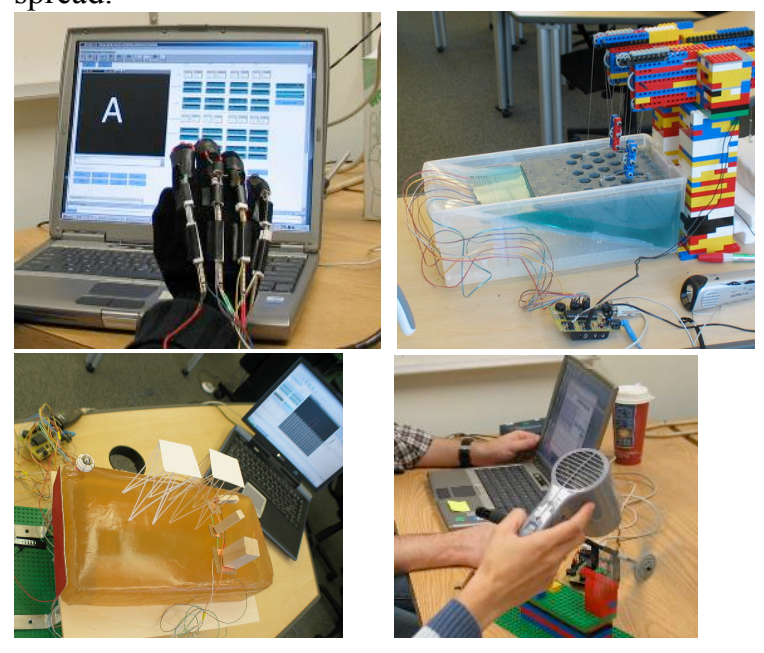

Figure 3. An ASL recognition glove, and models for investigating Tsunami wave patterns, earthquakes waves, and fire spread.

A student building an acid-base reaction model started to get interested in calculating the real-world scale of the virtual chemical reaction, which involved only 100 molecules. After several calculations with Avogadro's number, he was startled by the orders of magnitude of difference, which completely changed his view on the limitations of the computer model.

In addition, students were more careful "inventing" coefficients. Students designing a fire spread model conducted complex calculations to convert the rotational speed of their anemometer to real wind speed, as they wanted their measure to be accurate. In opposition, students of the first group (no sensors or robotics), oftentimes resorted to "unexplained" coefficients to make the simulation run faster or accordingly to their previous expectations.

\section{Conclusion and future work}

Our data so far indicates that there are particular concepts which students of the second group were more attentive to: error, friction, energy loss, and precision. Consistently, they were also more careful choosing coefficients. The trans-media approach enabled students to rapidly investigate their hypotheses and observe alternative outcomes.

This modeling framework is apparently an appropriated solution for some kinds of investigation, especially when the aforementioned topics (energy loss, etc.) are relevant. We are currently designing a new set of user studies and, on the technical side, working on incorporating image and video processing as a data-gathering tool.

\section{References}

[6] U. Wilensky, "NetLogo." Evanston, IL: Center for Connected Learning and Computer-Based Modeling. http://ccl.northwestern.edu/netlogo, 1999.

[7] A. Sipitakiat, P. Blikstein, and D. P. Cavallo, "GoGo Board: Augmenting Programmable Bricks for Economically Challenged Audiences," presented at International Conference of the Learning Sciences, Los Angeles, USA, 2004. 